\title{
ОЦЕНКА ЭФФЕКТИВНОСТИ ЛЕЧЕНИЯ ХИРУРГИЧЕСКИХ БОЛЬНЫХ С УЧЕТОМ ПРИМЕНЕНИЯ МАЛОИНВАЗИВНЫХ ТЕХНОЛОГИЙ
}

\section{EVALUATION OF THE EFFECTIVENESS OF TREATMENT OF SURGICAL PATIENTS TAKING INTO ACCOUNT THE USE OF MINIMALLY INVASIVE TECHNOLOGIES}

\section{Volkov Yu. Vinnik Yu. Volkov V. Sakovich}

Summary. The desire to improve the efficiency of treatment of surgical patients is a leading direction in the development of surgery. Currently, the transition to minimally invasive surgical interventions is taking place in most clinics in the world, which can be explained by the better final clinical result, as well as a more profitable and economically justified approach to surgical treatment. The transition to more gentle and cost-effective surgical methods occurs primarily because ineffective medical care can lead to an unreasonable deterioration in the health indicators of patients who are directly affected - both in terms of their well-being and in terms of their satisfaction with the work of the health care system as a whole. ... In a broader sense, the inefficiency of one or another component of the health care system may become the reason why other patients will be denied treatment and health improvement, who, if resources were spent more efficiently, would receive the necessary medical services. This statement is true primarily for systems that operate within an assigned overall budget. More broadly, inefficiencies in the health care system can lead to a drain on resources from the best performing health sector.

Keywords: surgery, effective surgery, minimally invasive technologies.
Волков Даниил Юрьевич

К.м.н., врач-сердечно-сосудистый хирург, ФГБУ «Федеральный центр сердечно-сосудистой хирургии»,

Красноярск

Винник Юрий Семёнович

Д.м.н., профессор, ФГБОУ ВО «Красноярский государственный медицинский университет им. проф. В.Ф. Войно-Ясенецкого», Красноярск

Волков Юрий Михайлович

Д.м.н., профессор, ФГБОУ ВО «Красноярский государственный медицинский университет им. проф. В.Ф. Войно-Ясенецкого», Красноярск volkov_ym@mail.ru

Сакович Валерий Анатольевич Д.м.н. профессор, главный врач, ФГБУ «ФЦССХ» 2. Красноярск; ФГБУ «Федеральный чентр сердечнососудистой хирургии», Красноярск

Аннотация. Стремление к повышению эффективности лечения хирургических больных является ведущим направлением в развитии хирургии. В настоящее время переход к малоинвазивным оперативным вмешательствам происходит в большинстве клиник мира, что можно объяснить лучшим конечным клиническим результатом, а также более выгодному и экономически обоснованному подходу к оперативному лечению. Переход к более щадящим и экономически выгодным оперативным методам происходит прежде всего потому, что нерезультативная медицинская помощь может привести к необоснованному ухудшению показателей здоровья пациентов, которые напрямую затронуты — как с точки зрения их самочувствия, так и с точки зрения их удовлетворенности работой системы здравоохранения в целом. В более широком смысле неэффективность тех либо других составляющих системы здравоохранения может стать причиной, по которой в лечении и улучшении состояния здоровья будет отказано иным пациентам, которые в случае более рационального расходования ресурсов получили бы нужные медицинские услуги. Данное утверждение справедливо в первую очередь для систем, которые действуют в рамках закрепленного общего бюджета. В более широком смысле неэффективность работы системы здравоохранения может привести к оттягиванию ресурсов из самого результативного сектора медицины.

Ключевые слова: хирургия, результативная хирургия, малоинвазивные технологии.

отбор из альтернативных программ. Из-за интенсивного роста медицинских технологий особое значение приобретает оценка эффективности лечебно-диагностических мероприятий. Определенная роль отводится исследованиям сравнительной результативности работы учреждений здравоохранения и определенных специалистов (рис. 1). 


\section{Предотвращение и сокращение прямых и косвенных потерь}

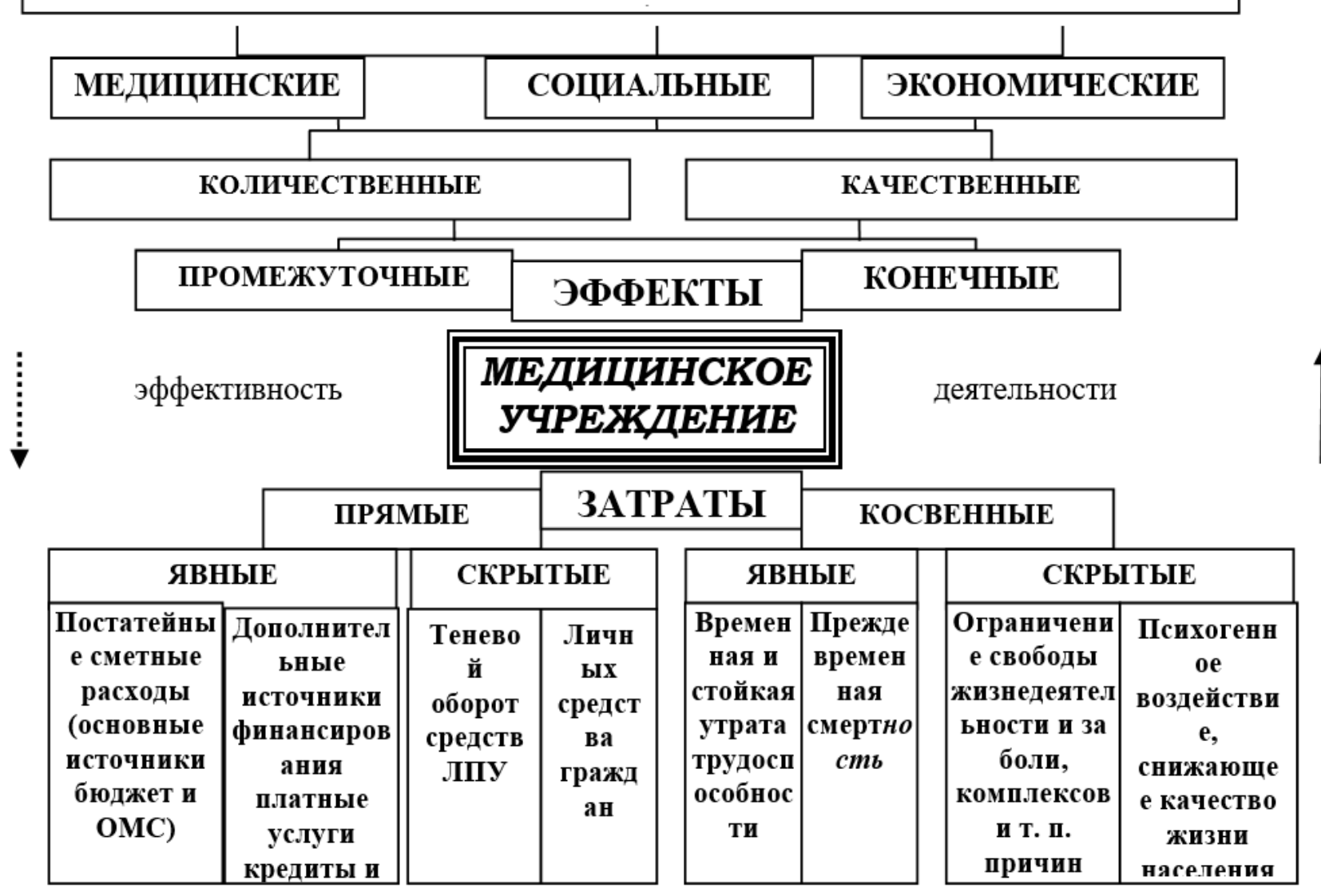

Рис. 1. Структура эффектов и затрат на рынке товаров и услуг здравоохранения

Эффективность - это степень достижения цели. Оценить эффективность, значит определить, насколько результат мероприятия, которое проводится или планируется, близок к желаемому.

Так, требования эффективности подразумевают такое соответствие структуры, а также функций, которое обеспечит получение самых высоких конечных результатов в сжатые сроки и с самыми малыми затратами сил и средств, либо укладывающиеся в рамки организационных задач. Чем больше частное от деления результатов на затраты, тем выше будет эффективность $[2,4,5]$. На данный момент, в эпоху развития миниинвазивных, видеоассистированых и эндоскопических операций, хирург стоит между сложным выбором того либо другого хирургического доступа. С одной стороны общепринятые принципы, которыми руководствуется хирург при выборе хирургического доступа, такими как анатомической и физиологической доступности, технической возможности, а также адекватной ревизии заинтересованных органов, и простоте исполнения для хирурга, а с другой стороны косметичность и атравматичность доступа, быстрая реабилитация и качество жизни после операции [6].
Несмотря на мировую тенденцию к минимизации хирургического доступа, до сих пор одним из наиболее часто используемых доступов к сердцу и органам средостения является полная продольная срединная стернотомия $[7,8,16]$. Использование срединной стернотомии обеспечивает хорошую визуализацию органов переднего средостения, удобство для хирурга и возможность расширения доступа при развитии осложнений. Такой доступ применяется при ишемической болезни сердца, приобретенных пороках аортального и митрального клапанов.

Однако в современных условиях применяются малоинвазивные доступы к сердцу, такие как министернотомия - при приобретенном пороке аортального клапана и торакотомия - при ишемической болезни сердца и приобретенном пороке митрального клапана. Принято говорить об эффективности малоинвазивных технологий.

Анализ эффективности, как правило, проводят с позиции "учреждения" и "программы" что соответствует анализу продуктивности и эффективности затрат [12]. 


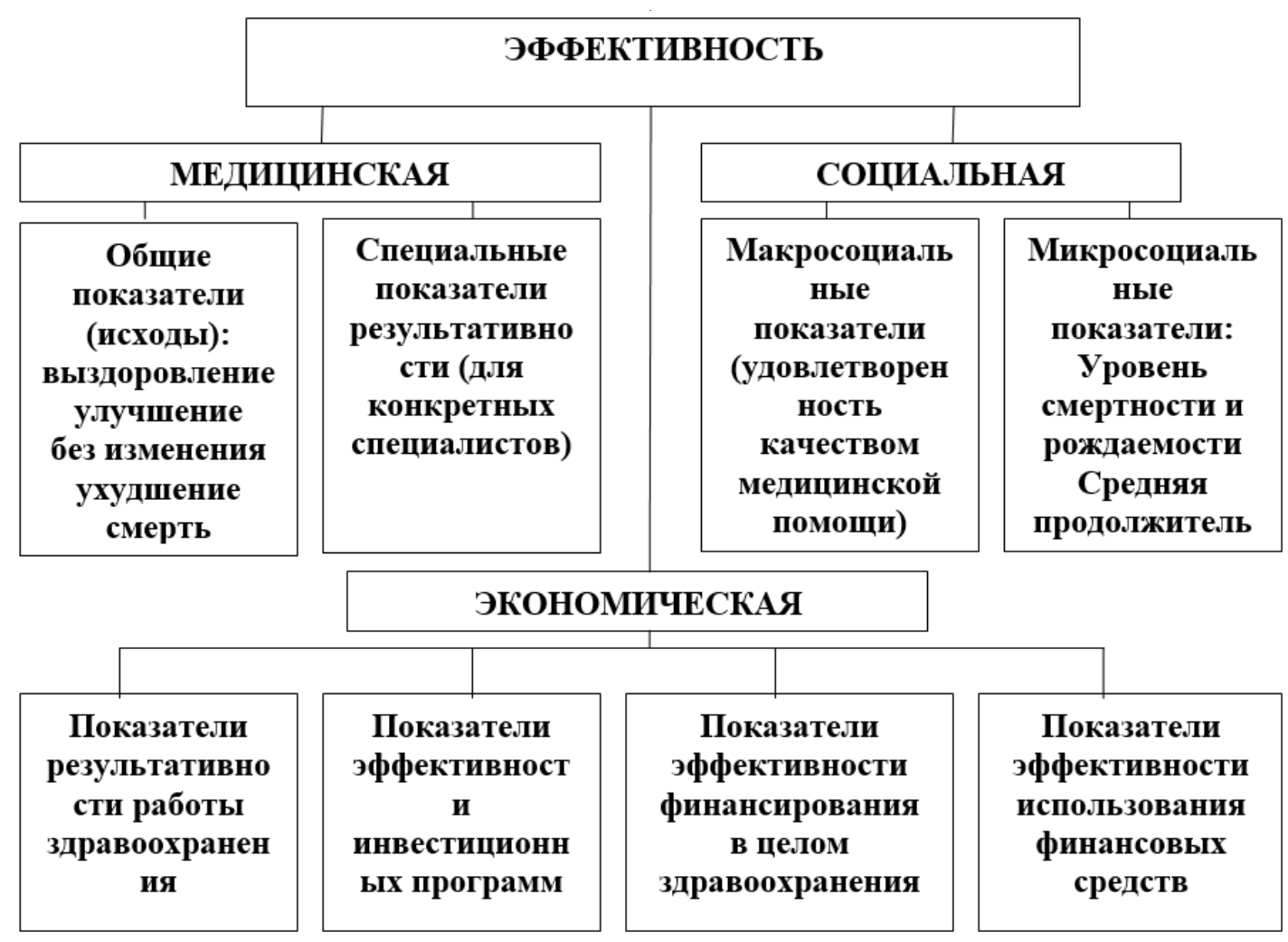

Рис. 2. Виды эффективности в здравоохранении

В оценке работы ЛПУ используется медицинская, социальная и экономическая эффективность.

Центр медико-социальных исследований и подготовки кадров Саратовского государственного медицинского университета в учебно-методическом пособии «Контроль качества медицинской помощи» предлагает следующую схему (1997) и описание к ней для вышеуказанных видов эффективности (рис. 2).

Для оценки медицинской эффективности используют два рода показателей:

- общие показатели;

- специальные показатели.

Под общими показателями понимают оцениваемые статистически показатели исходов: выздоровление, улучшение, без изменения; ухудшение, смерть.

Специальные показатели отражают только возможности оказания медицинской помощи внутри каждой конкретной медицинской специальности, например, хирургия - показатели послеоперационной летальности, для онкологов это выживаемость после курса лечения и т.д.

Показатели социальной эффективности можно разделить на две группы:
- микросоциальные показатели (показатели удовлетворенности качеством медицинской помощи);

- макросоциальные показатели (уровень смертности, рождаемости, средней продолжительности жизни и т.д.), с помощью которых определяются результаты работы системы здравоохранения по социально-демографическим показателям.

Социальную эффективность характеризуют рост продолжительности жизни, снижение заболеваемости, снижение преждевременной смертности в расчете на вес или обслуживаемое население территорий. В целом социальную эффективность как достижение определенных результатов здоровья оценивают по критерию полезности затрат $[9,10,11,12]$.

Единым недостатком для общих и стандартизованных показателей смертности является то, что они смещают значимость проблем здоровья в сторону старших возрастов. Так, основной причиной смерти населения РФ являются заболевания сердечно-сосудистой системы, в то же время средний возраст умерших превышает показатель продолжительности жизни на 10 лет.

Преодолеть указанные недостатки, в какой-то мере позволяют показатели потенциальной демографии [13, $17]$. 
Потерянные годы потенциальной жизни - показатель, характеризующий число лет жизни, потерянных вследствие преждевременной смертности и связанные с этим экономические последствия для региона или страны. Этот показатель может быть рассчитан для каждой болезни и, следовательно, использоваться для определения приоритетов в здоровье населения.

Bсе перечисленные показатели отражают только количество потерянных жизней или лет. В тоже время многие болезни не приводят непосредственно к смерти, но ухудшают качество жизни. Соответственно для определения приоритетов в выборе того или иного метода лечения необходим показатель, который отражал бы глобальное бремя болезни, объединяя потери вследствие преждевременной смертности и стойкой и временной нетрудоспособности, скорректировав их на качество жизни или степень трудоспособности.

Существуют две концепции, основанные на указанных принципах (Корчагин В.П., 1997).

QALY (Quality Adjusted Life Years) - годы жизни, скорректированные по качеству.

DALY - (Disability Adjusted Life Years) - годы жизни, скорректированные по нетрудоспособности.

Концепция QALY стала стандартным инструментом В оценке программ укрепления здоровья в середине восьмидесятых годов. В этом подходе основное внимание уделяется оценке с помощью сложных и дорогостоящих методов, потерь индивидуумом скорректированной по качеству жизни в связи со снижением физического умственного или социального статуса в течение определенного времени из-за различных причин.

Во избежание сложных и дорогостоящих процедур, связанных с расчетами индекса QALY, Мировым банком и Всемирной организацией здравоохранения был разработан другой подход к измерению глобального бремени болезней - DALY. Главная идея концепции DALY состоит в попытке качественной интегральной оценки состояния здоровья, принимая во внимание различные социальные роли в разных возрастах и их изменения с течением времени. Неотъемлемой частью концепции DALY является учет с помощью специальных коэффициентов, весомых социальных предпочтений состояния здоровья различных половозрастных групп населения.

Таким образом, используя концепцию расчетов QALY (Quality Adjusted Life Years) - годы жизни, скорректированные по качеству и DALY - (Disability Adjusted Life Years) - годы жизни, скорректированные по нетрудоспособности можно судить о преимуществах того или иного метода лечения с учетом длительности лечения, реабилитации и в, последующем, качестве жизни пациента. Это особенно актуально для определения экономической эффективности медицинского учреждения.

\section{ЛИТЕРАТУРА}

1. Вартанян Ф.Е., Сомин М.П. Вопросы экономической оценки лечебно-диагностических мероприятий // Здравоохр. Рос. Федерации.- 1991.№ 11.- C. 7-10.

2. Гиляревский С.Р. Оценка качества жизни больных ревматическим митральным пороком сердца после протезирования митрального клапана: Автореф. Дис. ... канд. мед. наук. - М., 1992.

3. Кохан Е.П. Оптимизация диагностики, лечения и профилактики инфекционных осложнений стернотомной раны у кардиохирургических больных / Е.П. Кохан, О.Н. Асанов, В.А. Потапов // Материалы 3 Международного конгресса «Раны и раневые инфекции», Москва.— 2016. — C. $172-174$.

4. Шейшенов, Ж.0. (13) Миниинвазивная хирургия сердца / Ж.0. Шейшенов // Современные тенденции развития науки и технологии.- 2016.№ 4-3.- - . 53-57.

5. Друкер П. Управление направленное на результаты. Пер. с англ. — М.: Технологическая школа бизнеса, 1994.

6. Жданов А.М. Экономический аспект перевода лечебно-профилактический учреждений Москвы на интенсивный способ развития // Экономика здраво0хр. - 1997. — №6.- С. 23. - 25

7. Жуков В.А., Скворцов А.Ф. Стационарная помощь: медико-экономические тенденции и перспективы развития // Экономика здравоохр.№ 6. - C. 12-19.

8. Кадыров Ф.Н. Экономические механизмы снижения госпитализации // Проблемы соц. гигиены и истории мед. - 1997.— № 2.— С. 28-31.

9. Калининская А.А. Экономический эффект от рационализации использования коечного фонда стационаров //Экономика здравоохр.- 2001.№ 3.- С. 23-26

10. Кравченко Н.А., Поляков И.В. Научное обоснование методологии прогнозирования ресурсного обеспечения здравоохранения России. М., 1998.-392 с.

11. Корчагин В.П. Финансовое обеспечение здравоохранения / М-во науки и технологий РФ, 1997. — 45 c.

12. Кулагина Э.Н. Введенская И.И. Многовариантность критериев оценок экономической эффективности в здравоохранении. Здравоохранение РФ.1998, № 5. ( 36-39

13. Лисицын Ю.П. «Модус» здоровья Россиян // Экономика здравоохр. — 2001.— № 2.— С. 32-37 
14. Самородская И.В. Научно обоснованная экономика здравоохранения //Экономика здравоохр. — 2001. — № 3. - C. 18-20

15. Conviser, Richard. "A Brief History of the Oregon Health Plan and its Features.", June 1997.

16. Almdahl, S.M. Avoidance of noninfectious sternal dehiscence: figureof-8 wiring is superior to straight wire closure / S.M. Almdahl, P. Halvorsen, T. Veel, S.E. Rynning // Scand Cardiovasc J. — 2013. — Vol. 47, — P. 247-250.

17. Falagas M.E. Impact of vacuum-assisted closure (VAC) therapy on clinical outcomes of patients with sternal wound infections: a meta-analysis of nonrandomized studies / M.E. Falagas, G.S. Tansarli, A. Kapaskelis, K.Z. Vardakas // PLoS One. — 2013. — Vol. 8, — P.74-86.

18. Vogt P.R. (21) Significant reduction of sternal wound infection in cardiac surgical patients / P.R. Vogt, A. Darral, P.A. Berdat [et al.] // Бюллетень НЦССХ им. А.Н. Бакулева РАМН. Сердечно-сосудистые заболевания. — 2019. — № 4. - Том 20. — С. 368-378.

( С Волков Даниил Юрьевич, Винник Юрий Семёнович (volkov_ym@mail.ru ),

Волков Юрий Михайлович, Сакович Валерий Анатольевич.

Журнал «Современная наука: актуальные проблемы теории и практики»

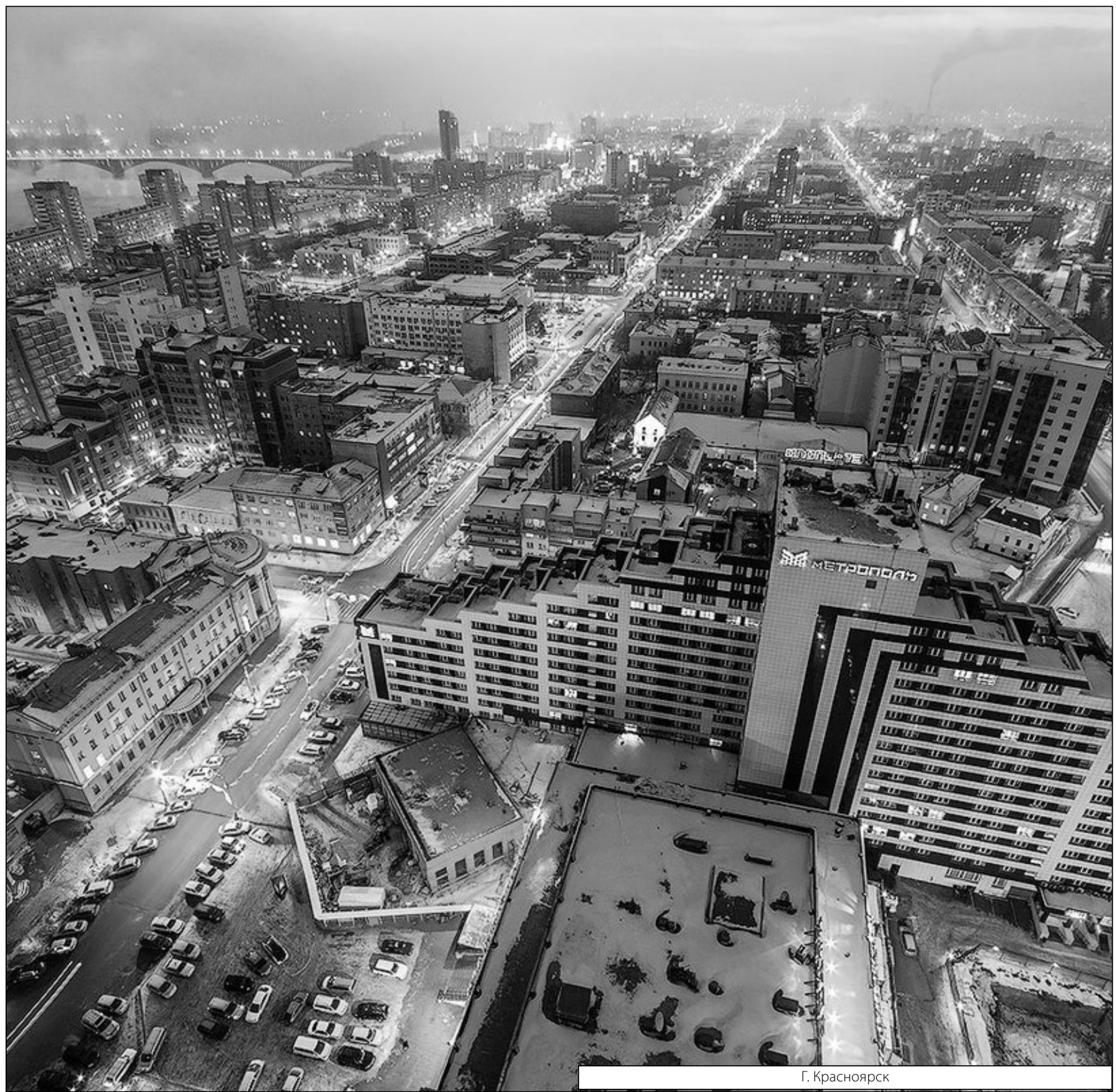

